$12,11,03,09$

\title{
Двумерные триплетные магнитоэкситоны и магнетофермионный конденсат в GaAs/AIGaAs гетероструктурах
}

\author{
(ㄱ Л.В. Кулик, А.В. Горбунов, А.С. Журавлев, В.Б. Тимофеев \\ Институт физики твердого тела РАН, \\ Черноголовка, Россия \\ ฯ E-mail: timofeev@issp.ac.ru
}

В двумерном холловском изоляторе с высокой подвижностью электронов, фактором заполнения $v=2$ и температурах $T<1 \mathrm{~K}$ при фотовозбуждении достаточно плотного газа долгоживущих триплетных циклотронных магнитоэкситонов обнаружено принципиально новое коллективное состояние - магнетофермионный конденсат. Конденсированная фаза взаимодействует когерентно с внешним электромагнитным полем, демонстрирует сверхизлучающие свойства при рекомбинации коррелированных электронов конденсата с тяжелыми дырками валентной зоны, а также бездиссипативно растекается в слое двумерного электронного газа на макроскопически большие расстояния, перенося целочисленный спин. Обнаруженные эффекты объясняются в терминах когерентного конденсата в неравновесной системе двумерных фермионов с полностью квантованным энергетическим спектром, в котором присутствует вырожденный ансамбль долгоживущих триплетных магнитоэкситонов, подчиняющихся бозевской статистике.

Работа поддержана Российским научным фондом (грант № 16-12-10075).

DOI: $10.21883 /$ FTT.2018.08.46255.01Gr

\section{1. Введение}

В физике конденсированных сред принято считать, что наиболее яркие коллективные явления связаны с бездиссипативным, сверхтекучим течением материи электрически заряженной (сверхпроводники) или электрически нейтральной $\left({ }^{4} \mathrm{He},{ }^{3} \mathrm{He}\right)[1]$. В случае сверхтекучего ${ }^{4} \mathrm{He}$, где гелиевые атомы непосредственно являются бозе-частицами, явление бездиссипативного течения непосредственно связано с явлением бозеэйнштейновской конденсации в сильно неидеальном бозе-газе взаимодействующих атомов ${ }^{4} \mathrm{He}$. Сверхпроводники и жидкость изотопа ${ }^{3} \mathrm{He}$ являются фермиевскими объектами. Ключевую роль в бездиссипативном течении материи в этих объектах играют связанные пары фермиевских частиц, которые являются композитными бозонами, а именно: куперовские электронные пары в сверхпроводниках и спин-коррелированные пары изотопов атомов ${ }^{3} \mathrm{He}$ в электрически нейтральной фермиевской ${ }^{3}$ Не-жидкости. При достижении критических условий по плотности и температуре ансамбль бозе-частиц (или квазичастиц) конденсируется в фазовом пространстве, образуя коллективное когерентное состояние - бозе-эйнштейновский конденсат. Существенно, что во всех перечисленных выше объектах коллективные явления протекают в условиях детального термодинамического равновесия. Главная мотивация наших исследований состояла в попытке ответить на вопрос, могут ли в фермиевской системе происходить коллективные явления в термодинамически неравновесных, но близких к квазиравновесным, условиях, когда в такой системе тем или иным способом, например с помощью фотовозбуждения при низких температуpax, создается подсистема электрически нейтральных бозе-частиц достаточной плотности. Нам представляется, что среди разнообразных по своей природе нейтральных бозе-возбуждений (например, плазмонов, магнонов и др.) экситоны, обладающие бозевской природой, являются наиболее предпочтительным физическим объектом.

Интерес к экситонным возбуждениям в фермиевских системах носителей заряда возник достаточно давно. В этой связи уместно напомнить о сингулярности Махана, которая возникает на краю вырожденного фермиевского спектра в легированном полупроводнике из-за кулоновских электронно-дырочных корреляций (см., например, работу [2]). Другим примером могут служить рентгеновские экситоны, впервые исследованные в теоретической работе [3]. В случае возбуждения экситонов в трехмерных металлах, в частности рентгеновских экситонов, считалось, что такие неравновесные состояния крайне нестабильны и являются короткоживущими. Действительно, в недавно выполненных экспериментах c использованием фемтосекундной лазерной техники возбуждались очень короткоживущие экситоны на поверхности металлического серебра [4]. Однако ситуация с временами жизни экситонных возбуждений определенного типа в двумерном (2D) металле, помещенном в достаточно сильное поперечное слою магнитное поле, о которых пойдет речь в данной статье, изменяется самым радикальным образом. Принципиальная возможность возбуждения экситонных состояний в режиме квантового эффекта Холла, целочисленного и дробного, уже обсуждалась в литературе [5-7], равно как и сама возможность существования долгоживущих экситонных возбуждений [8-10]. Отметим также теоретическую работу [11], в которой показано, что в сильном магнитном 
поле двумерная, электрически нейтральная электроннодырочная система должна вести себя почти как идеальный бозе-газ.

\section{2. Магнитоэкситоны в двумерном холловском изоляторе}

Нами были исследованы (см. работу [12]) 2D-экситоны в условиях фотовозбуждения холловского изолятора при факторе заполнения $v=2$ в широких (17 и $35 \mathrm{~nm}$ ), симметрично легированных квантовых ямах (КЯ) в высокосовершенных $\mathrm{GaAs} / \mathrm{AlGaAs-}$ гетероструктурах с темновой подвижностью электронов $\mu_{e}=(5-20) \cdot 10^{6} \mathrm{~cm}^{2} / \mathrm{V} \cdot \mathrm{c}$ и концентрациями $n_{e}=(0.5-2.5) \cdot 10^{11} \mathrm{~cm}^{-2}$. Все эксперименты выполнялись в специально изготовленной криосистеме, размещенной в сверхпроводящем соленоиде, с изолированной камерой с жидким ${ }^{3} \mathrm{He}$, в которой размещались исследуемые образцы, световоды, поляризаторы, фильтры, а также была предусмотрена откачка гелиевых паров для достижения температур вплоть до $0.45 \mathrm{~K}$. Для подвода к образцу возбуждающего лазерного излучения и сбора светового отклика от образца (отражения, неупругого рассеяния света, люминесценции) использовалась световодная техника.

В настоящей работе двумерные электроны основного, полностью заполненного уровня Ландау $\left(n_{L}^{e}=0\right)$ возбуждались с помощью межзонной резонансной лазерной накачки на ближайший электронный циклотронный уровень $\left(n_{L}^{e}=1\right)$. В таких условиях на основном электронном циклотронном уровне $\left(n_{L}^{e}=0\right)$ появляются ферми-дырки (вакансии электронов). Фотовозбужденные электроны связываются с положительно заряженными ферми-дырками и образуют экситоны подобно тому, как рождаются светом экситоны в обычном собственном полупроводнике. Такие экситоны будем в дальнейшем называть циклотронными магнитоэкситонами (ЦМЭ). Благодаря спиновым степеням свободы электронов и дырок ЦМЭ оказываются двух типов: синглетные и триплетные (рис. 1). У синглетных экситонов (СЦМЭ) результирующий спин $S=0$, и их энергия возбуждения в силу высокой электронно-дырочной симметрии, согласно теореме Кона [13], в точности равна циклотронной энергии, а энергия связи равна нулю. Поэтому СЦМЭ называют еще и бесспиновым магнитоплазмоном. Радиационное время жизни синглетного магнитоэкситона определяется дипольно-разрешенной рекомбинацией электрона первого циклотронного уровня $\left(n_{L}^{e}=1\right)$ с ферми-дыркой на основном электронном уровне $\left(n_{L}^{e}=0\right)$ с испусканием циклотронного фотона. Характерные времена такой излучательной рекомбинации составляют доли наносекунды [14]. У триплетных циклотронных магнитоэкситонов (ТЦМЭ) полный спин $S=1$, а спиновые проекции вдоль направления магнитного поля принимают значения $S_{z}=-1,0,+1$. Такие экситоны рождаются только с опрокидыванием спина электрона (spin-flip)

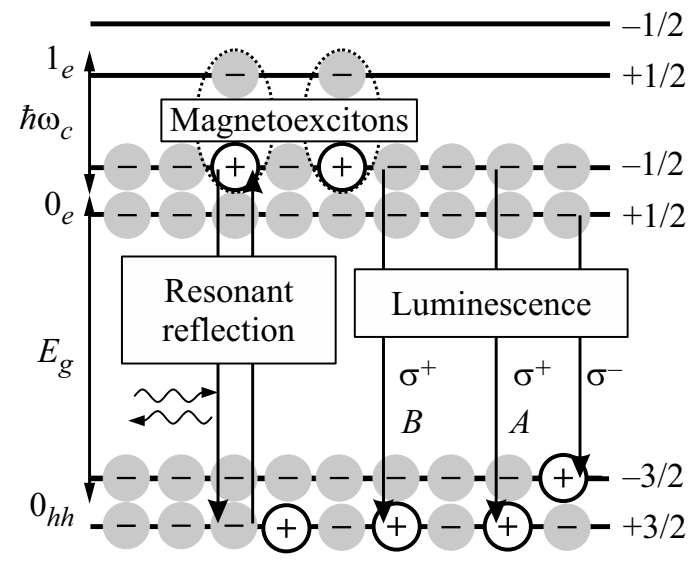

Рис. 1. Одноэлектронная схема энергетических состояний нулевого, $0_{e}$, и первого, $1_{e}$, уровней Ландау $2 \mathrm{D}$-электронов зоны проводимости, а также нулевого, $0_{h h}$, уровня Ландау тяжелых дырок валентной зоны $( \pm 1 / 2, \pm 3 / 2-$ спиновые состояния электронов и тяжелых дырок соответственно). Схематически показано, как в условиях резонансной оптической накачки возникающие на нулевом уровне Ландау ферми-дырки (электронные вакансии) могут связываться с возбужденными электронами на первом уровне Ландау, образуя ТЦМЭ. Вертикальные стрелки слева - оптические переходы между уровнями тяжелых дырок и нулевым уровнем Ландау электронов в условиях резонансного отражения. Вертикальные стрелки справа - излучательные переходы между одноэлектронными состояниями в двух циркулярных поляризациях: $\sigma^{-}$и $\sigma^{+}(A)$. При образовании плотного ансамбля ТЦМЭ, доминирующим становится переход $\sigma^{+}(B)$.

при возбуждении электрона из основного на ближайший уровень Ландау. В отличие от СЦМЭ, которые называют еще „светлыми“ (bright), ТЦМЭ являются „темными“ (dark), поскольку их прямое возбуждение и излучательная аннигиляция строго запрещены в дипольном приближении. Вместе с тем для триплетных экситонов не возникают ограничения из-за соображений симметрии, связанные с теоремой Кона. Поэтому они обладают энергией связи, обусловленной кулоновским электронно-дырочным взаимодействием. Заметим, что синглет-триплетное расщепление обращается в ноль при импульсе $k=0$ только в первом порядке теории возмущений по малому параметру отношения кулоновской энергии к циклотронной. Во втором порядке теории возмущений в рассматриваемых магнитных полях это расщепление достигает долей $\mathrm{meV}$ [15]. Именно это расщепление приводит к тому, что ТЦМЭ оказывается возбуждением с наименьшей энергией при всех четных целочисленных состояниях квантового эффекта Холла [8-9].

Возбуждение ТЦМЭ с помощью прямых резонансных оптических переходов строго запрещено в дипольном приближении, равно как строго запрещена их прямая излучательная аннигиляция. Отсюда возникают трудности их прямого оптического детектирования и кон- 
тролируемого манипулирования их плотностью. В этой связи нами отработана экспериментальная техника [12], с помощью которой можно возбуждать светом ансамбль трансляционно-инвариантных, т.е. свободных, ТЦМЭ, контролировать их плотность и исследовать кинетику релаксации в основное состояние (речь идет об измерениях безызлучательного времени жизни триплетных ЦМЭ). Предложенный метод основан на измерениях резонансного отражения в результате прямых межзонных дипольно-разрешенных переходов между дискретными состояниями (уровнями Ландау) тяжелых дырок валентной зоны и дискретными состояниями электронов (уровнями Ландау) в зоне проводимости. Априори можно предполагать, что в условиях тестирования холловского изолятора при факторе заполнения $v=2$ сигнал резонансного отражения, соответствующий прямому переходу из нулевого уровня Ландау тяжелых дырок валентной зоны $\left(n_{L}^{h h}=0\right)$ на нулевой уровень Ландау электронов зоны проводимости $\left(n_{L}^{e}=0\right)$, должен отсутствовать, поскольку все электронные состояния основного электронного циклотронного уровня полностью заполнены. Однако при включении оптической накачки, которая возбуждает электроны посредством дипольно-разрешенных межзонных резонансных переходов на верхние уровни Ландау $\left(n_{L}^{e}>1\right)$, при достаточно низкой температуре естественно ожидать образования ТЦМЭ с проекцией спина $S_{z}=-1$, поскольку именно эти экситоны являются возбужденным состоянием с наименьшей энергией в фотовозбужденной 2D-электронной системе в магнитном поле. Поэтому процесс формирования ТЦМЭ должен проявиться в уменьшении числа незаполненных состояний первого электронного уровня Ландау $\left(n_{L}^{e}=1\right)$ и в одновременном возникновении незаполненных состояний (ферми-дырок) на нулевом электронном уровне $\left(n_{L}^{e}=0\right)$. Соответствующие изменения в спектрах отражения естественно назвать фотоиндуцированным резонансным отражением (ФРО). В спектрах ФРО должны наблюдаться два резонансных пика: положительный пик, соответствующий оптическому межзонному переходу (0-0) с нулевого циклотронного уровня тяжелых дырок $n_{L}^{h h}=0$ на верхний спиновый подуровень нулевого циклотронного уровня двумерных электронов $n_{L}^{e}=0$, и отрицательный пик, отвечающий переходу $(1-1)$ с первого циклотронного уровня тяжелых дырок валентной зоны $n_{L}^{h h}=1$ на первый электронный циклотронный уровень $n_{L}^{e}=1$. Если положительный пик ответствен за возникновение и рост с накачкой числа ферми-дырок на верхнем спиновом подуровне нулевого циклотронного уровня электронов $n_{L}^{e}=0$, то отрицательный пик отвечает за уменьшение с ростом накачки числа дырок на первом циклотронном электронном уровне $n_{L}^{e}=1$. Таким образом, предлагаемый метод ФРО является непрямым способом тестирования триплетных (оптически неактивных!) ЦМЭ с использованием оптически разрешенных переходов из валентной зоны в зону проводимости переходы $(0-0)$ и $(1-1)$.

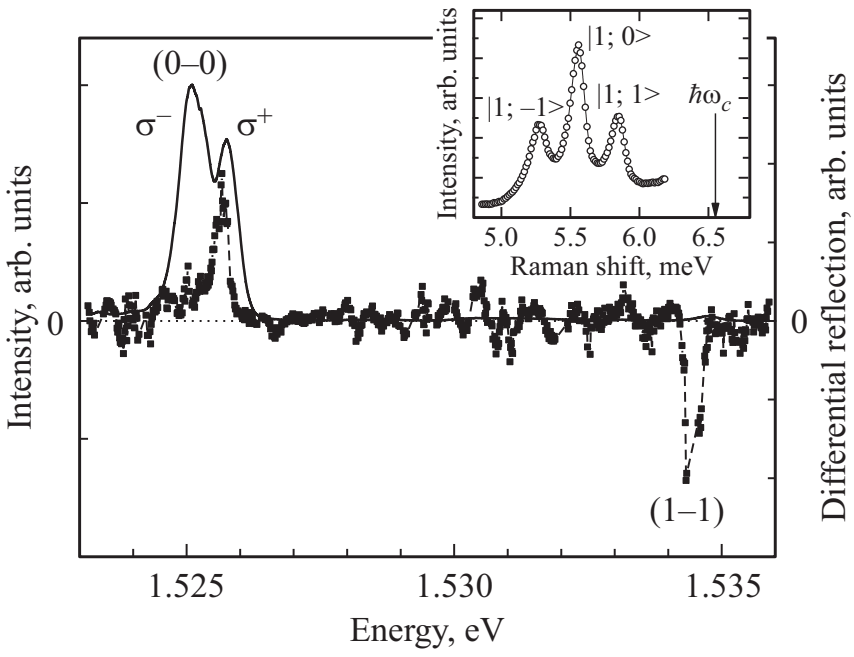

Рис. 2. Спектры ФЛ (сплошная линия) и ФРО (точки и пунктирная линия) при $v=2$, измеренные в КЯ $\mathrm{GaAs} / \mathrm{AlGaAs}$ шириной $17 \mathrm{~nm}\left(\mu_{e}=5 \cdot 10^{6} \mathrm{~cm}^{2} / \mathrm{V} \cdot \mathrm{s}, n_{e}=2.4 \cdot 10^{11} \mathrm{~cm}^{-2}\right)$ в перпендикулярном плоскости ямы магнитном поле $5 \mathrm{Tl}$ при температуре $T=0.45 \mathrm{~K}$. Оптический переход $(0-0)$ происходит между основными циклотронными уровнями электронов зоны проводимости $n_{L}^{e}=0$ и тяжелых дырок валентной зоны $n_{L}^{h h}=0$, переход $(1-1)-$ между первыми циклотронными уровнями: $n_{L}^{e}=1$ и $n_{L}^{h h}=1$. На вставке - спектр резонансного неупругого рассеяния света, измеренный при тех же экспериментальных условиях и дополнительной параллельной компоненте магнитного поля $5 \mathrm{~T}$, введенной для усиления расщепления спиновых компонент экситонного триплета.

Выполненные эксперименты полностью подтверждают изложенную выше суть метода ФРО. По спектрам ФРО (см. рис. 2) удавалось контролировать рождение фотовозбужденных электронов на первом циклотронном уровне $\left(n_{L}^{e}=1\right)$ и ферми-дырок на нулевом циклотронном уровне 2D-электронной системы $\left(n_{L}^{e}=0\right)$. На рис. 2 представлены также спектры фотолюминесценции (ФЛ), измеренные при факторе заполнения $v=2$ в тех же условиях, при которых измерялись спектры ФРО. В спектре ФЛ отчетливо виден циркулярнополяризованный дублет $\left(\sigma^{+}\right.$и $\sigma^{-}$-компоненты, соответствующий электронным переходам $(0-0)$. Величина дублетного расщепления соответствует сумме спинового расщепления в магнитном поле основного циклотронного уровня тяжелых дырок валентной зоны $n_{L}^{h h}=0$ и спинового расщепления основного циклотронного уровня двумерных электронов зоны проводимости $n_{L}^{e}=0$. В спектре $Ф Р О$, в полном соответствии с изложенными выше ожиданиями, виден положительный пик в области электронных переходов (0-0), а также отрицательный пик в области электронных переходов $(1-1)$. С ростом мощности накачки положительный пик вырастает, а отрицательный по интенсивности падает: поведение интенсивностей этих пиков имеет самосогласованный характер. Такое поведение спектров ФРО обусловлено образованием низкоэнергетических ТЦМЭ, состоящих 

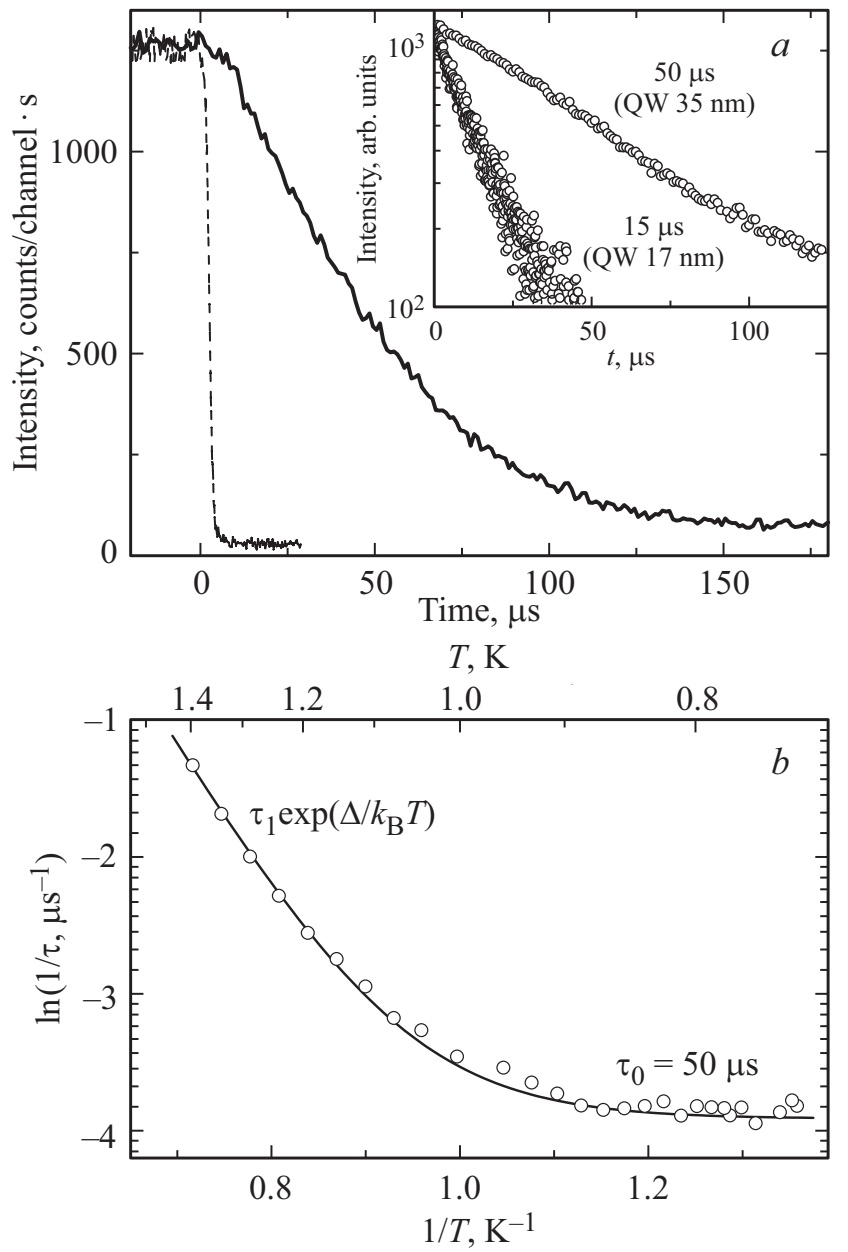

Рис. 3. (a) Пример кинетики затухания ФРО (сплошная кривая) и для сравнения аппаратная функция измерительной системы (пунктир). На вставке в полулогарифмическом масштабе - кинетика затухания, измеренная для КЯ с ширинами 35 и $17 \mathrm{~nm}$ при $v=2$ в перпендикулярном магнитном поле $B=4$ T. В обеих КЯ $n_{e}=2 \cdot 10^{11} \mathrm{~cm}^{-2}$, и соответственно $\mu_{e}=15 \cdot 10^{6}$ и $5 \cdot 10^{6} \mathrm{~cm}^{2} / \mathrm{V} \cdot \mathrm{s}$. (b) Температурная зависимость скорости релаксации ТЦМЭ $1 / \tau$ в логарифмическом масштабе в КЯ шириной $35 \mathrm{~nm}$ в магнитном поле $4 \mathrm{~T}$. Точки - эксперимент, линия - аппроксимация с учетом двух механизмов релаксации: активационного в области $T>1 \mathrm{~K}$ и не зависящего от температуры в области $T<1 \mathrm{~K}$.

из электронов первого электронного уровня Ландау $n_{L}^{e}=1$, связанных с ферми-дырками нулевого электронного уровня $n_{L}^{e}=0$ (см. диаграмму переходов на рис. 1$)$. Подчеркнем, что возбуждение ТЦМЭ происходит в вырожденной фермиевской электронной системе.

Несмотря на то что триплетные ЦМЭ не являются оптически активными, их существование можно установить прямым образом по спектрам неупругого рассеяния света (см. вставку на рис. 2) и с их помощью непосредственно определить величину синглет-триплетного расщепления экситонов $[12,15]$. Синглет-триплетное экситонное расщепление измеряется как разность энергий синглетного экситона и центра тяжести экситонного триплета (положения в спектре спиновой компоненты $\left.S_{z}=0\right)$, наблюдаемого в спектрах неупругого рассеяния света. Это расщепление, которое и определяет энергию связи триплетного экситона, оказалось сравнительно большим, около $1 \mathrm{meV}$ [12].

Использование техники ФРО в импульсном режиме оказалось очень эффективным для исследования времени спиновой релаксации $\tau$, т.е. времен жизни ТЦМЭ в зависимости от температуры $T$, величины магнитного поля $B$, ширины квантовой ямы (КЯ), и что очень важно, для установления качества самих гетероструктур. Поскольку времена релаксации триплетных магнитоэкситонов оказались чрезвычайно большими и в самых высококачественных структурах эти времена достигали масштабов миллисекунды, для измерений столь больших времен использовалась простая техника механического прерывателя (вращающийся металлический диск с узкой щелью) [12]. На рис. 3, $a$ в качестве иллюстрации приведены результаты измерения кинетики затухания импульсного сигнала ФРО, соответствующего безызлучательному распаду ТЦМЭ. В температурной зависимости времени релаксации $\tau(T)$, представленной на рис. $3, b$ в полулогарифмическом масштабе, можно видеть две области различного температурного поведения: активационную область $(T>1 \mathrm{~K})$ и область, в которой зависимость от температуры отсутствует $(T<1 \mathrm{~K})$. Активационная область связана с процессами тепловой диссоциации ТЦМЭ, в результате которой рождаются свободные электрон и ферми-дырка, которые, в конечном итоге, рекомбинируют с испусканием циклотронного фотона. Другая температурная область, где время спиновой релаксации перестает зависеть от температуры, определяется исключительно качеством гетероструктуры: выше качество - длиннее время спиновой релаксации. Существенный вклад в выяснение механизма релаксации ТЦМЭ привнесли теоретические расчеты С.М. Дикмана (см., например, [10]), в которых убедительно показано, что в высокосовершенных структурах основной механизм релаксации связан с процессом неупругого рассеяния ТЦМЭ на коротковолновых акустических фононах.

\section{3. Магнетофермионный конденсат: фазовая диаграмма}

Обнаруженные большие времена жизни ТЦМЭ в 2D-электронной системе, рекордные для трансляционноинвариантных систем, открыли уникальную возможность изучения при достаточно низких температурах вырожденной фермиевской системы, в которой с помощью света возбужден плотный ансамбль долгоживущих ТЦМЭ, подчиняющихся бозе-статистике. Ниже будут изложены наши основные экспериментальные наблюдения и на качественном уровне даны объяснения наиболее важным результатам [16]. 

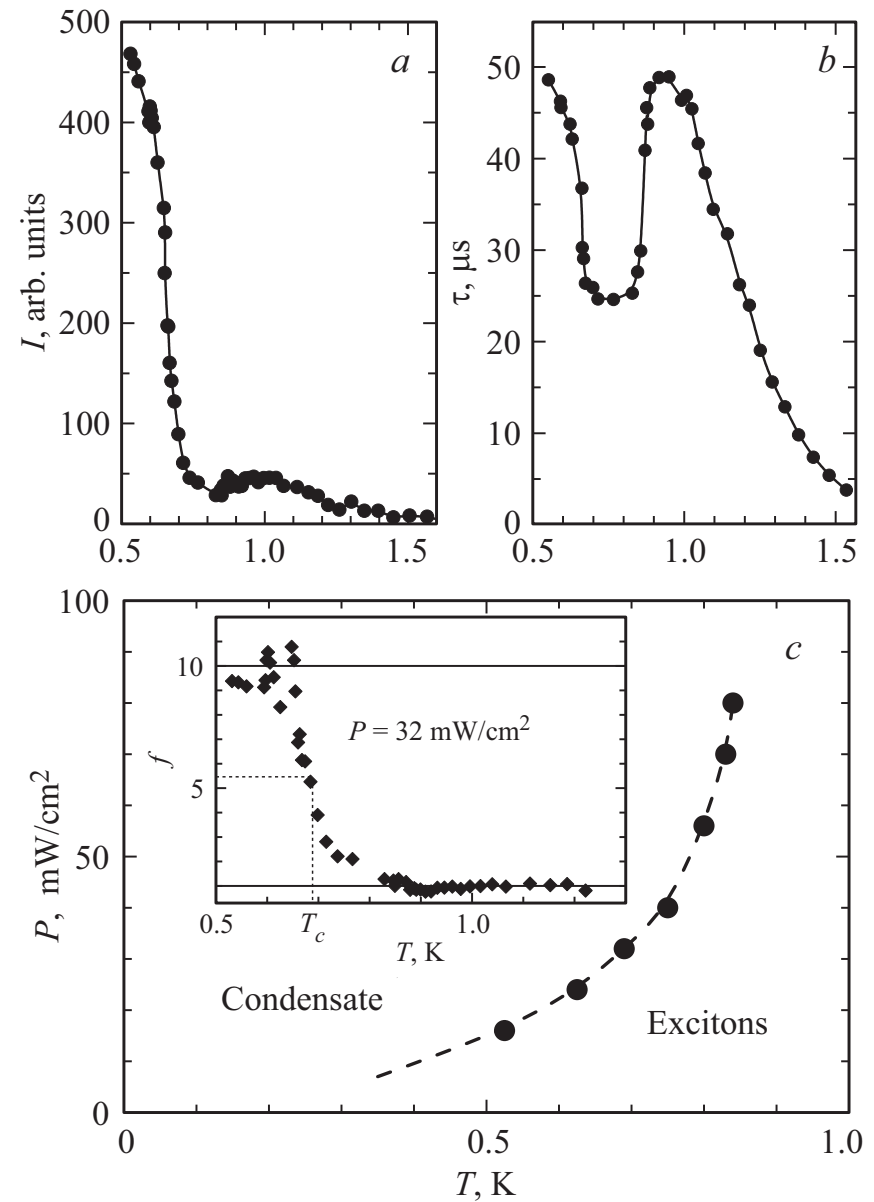

Рис. 4. (a) Температурная зависимость интенсивности сигнала ФРО $I(T)$. (b) Температурная зависимость времени релаксации ТЦМЭ $\tau(T)$. (c) Фазовая диаграмма конденсации ТЦМЭ: фазовая граница (точки - эксперимент, штриховая линия - аппроксимация) разделяет области газа магнитоэкситонов от конденсата. На вставке показано, как определялась критическая температура перехода $T_{c}$ : на половине высоты порогового возрастания силы осциллятора $f$.

Первый цикл экспериментов касался изучения ФРО при вариациях плотности мощности оптической накачки $P$ и температуры $T$. Остановимся сначала на измерениях дифференциала резонансного отражения в функции температуры при большой и фиксированной оптической накачке, создающей достаточно высокую плотность ТЦМЭ, $n_{\mathrm{ex}}=10^{10} \mathrm{~cm}^{-2}$ (см. рис. 4, $\left.a, b\right)$. Было обнаружено, что при заданной фиксированной накачке $\left(P \approx 30 \mathrm{~mW} / \mathrm{cm}^{2}\right)$ и понижении температуры в области $T \leq 0.8 \mathrm{~K}$ интенсивность резонансного отражения $I(T)$ пороговым образом возрастает более чем на порядок (рис. 4,a). Пороговый характер этого явления указывает на возникновение новой конденсированной фазы ТЦМЭ типа бозе-конденсата с заметно большей плотностью (большими числами заполнения). Это заключение подтверждают эксперименты, когда сравниваются температурные зависимости времени спиновой релаксации $\tau(T)$, которые определяют времена жизни ТЦМЭ, с дифференциалом резонансного отражения $I(T)$ при одной и той же накачке. В окрестности порога (в данном случае $T \approx 0.8 \mathrm{~K})$ и дальнейшем понижении температуры было установлено, что ФРО возрастает даже там, где времена жизни экситонов несколько уменьшаются (рис. 4,b). Порог возрастания ФРО оказался очень чувствительным к мощности оптической накачки и при уменьшении плотности мощности сдвигался в сторону более низких температур. Поведение порога возрастания дифференциала резонансного отражения в функции температуры позволяет экспериментально определить фазовую диаграмму с фазовой границей, которая разделяет области существования экситонного газа и новой, более плотной, конденсированной фазы ТЦМЭ. Такая экспериментально измеренная фазовая диаграмма представлена на рис. 4, $c$. Здесь точки соответствуют эксперименту, а проведенная по ним и продолженная к началу координат пунктирная линия служит исключительно курсором для глаза. На вставке к рис. 4, с продемонстрировано, как именно определялся порог возникновения новой экситонной фазы: по изменению интенсивности ФРО $I(T)$ в функции температуры при фиксированной оптической накачке. Отсчет критической температуры $T_{c}$ проводился на половине высоты ступеньки, наблюдаемой для параметра $f(T)=I(T) \tau(T)$, пропорционального силе осциллятора оптического перехода.

На рис. 4, $b$ для сравнения с интенсивностью ФРО приведено поведение времени жизни (времени спиновой релаксации) ТЦМЭ $\tau(T)$ при фиксированной накачке и вариации температуры. Согласно рис. $4, b$, время жизни триплетных магнитоэкситонов при уменьшении температуры и фиксированной накачке ведет себя немонотонно: сначала возрастает, что естественно, а затем начинает уменьшаться, что оказалось совершенно неожиданным. К обсуждению вопроса о немонотонном поведении времени жизни триплетных магнитоэкситонов мы вернемся несколько позже, в разд. 4.

Обратимся теперь к спектрам люминесценции, которые исследовались при различных мощностях фотовозбуждения и вариации температуры в условиях, когда возникает новая конденсированная фаза ТЦМЭ (см. рис. 5). Здесь следует напомнить, что излучательная аннигиляция триплетных ЦМЭ в дипольном приближении строго запрещена. Этот запрет в одинаковой мере касается и излучательного распада ТЦМЭ, оказавшихся в новом фазовом состоянии. Поэтому для нас стало полной неожиданностью появление нового, весьма интенсивного, канала люминесценции, обнаруженного в условиях рождения нового фазового состояния ТЦМЭ. Этот новый канал излучательной рекомбинации возникает и в конечном итоге становится доминирующим в спектральной области, соответствующей межзонной рекомбинации 2D-электронов нулевого уровня Ландау зоны проводимости с тяжелыми дырками основного уровня Ландау валентной зоны, согласно схеме одноэлектронных состояний (см. рис. 1). 


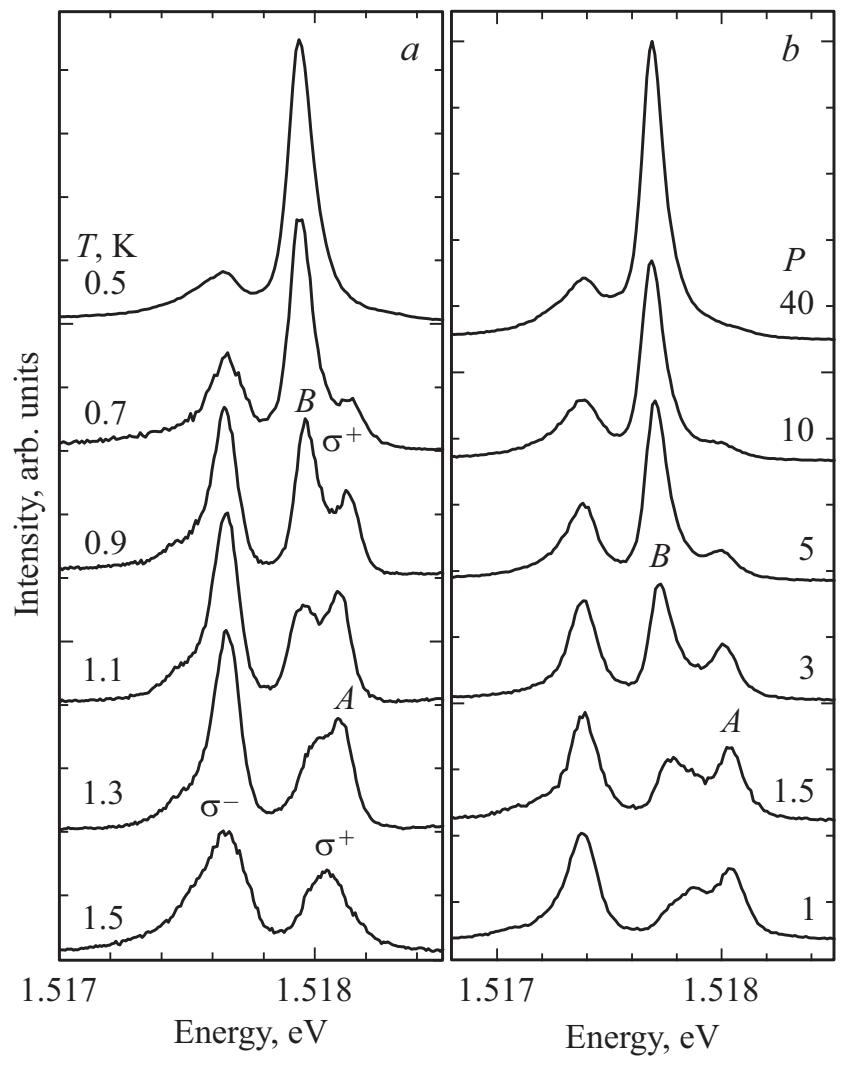

Рис. 5. Спектры люминесценции, соответствующие излучательной рекомбинации 2D-электронов ниже уровня Ферми в зоне проводимости с тяжелыми дырками в валентной зоне: (a) при различных температурах $T$, указанных около каждого спектра, и фиксированной накачке $P=32 \mathrm{~mW} / \mathrm{cm}^{2} ;(b)$ при $T=0.45 \mathrm{~K}$ и различных накачках, указанных около каждого спектра в $\mathrm{mW} / \mathrm{cm}^{2}$.

Рис. 5, $а$ демонстрирует, как изменяются соответствующие спектры ФЛ при вариации температуры в интервале $1.5-0.5 \mathrm{~K}$ и фиксированной накачке, $P \cong 32 \mathrm{~mW} / \mathrm{cm}^{2}$. При $T=1.5 \mathrm{~K}$, а также относительно слабых оптических накачках в спектрах люминесценции наблюдается известный циркулярно поляризованный дублет. Расстояние между компонентами дублета $\sigma^{+}-\sigma^{-}$, линии которого отличаются знаком циркулярной поляризации в соответствии со схемой дипольно-разрешенных оптических переходов (рис. 1), определяется суммой спиновых расщеплений 2D-электронов нулевого уровня Ландау и основного циклотронного уровня тяжелых дырок валентной зоны. Однако при понижении температуры и фиксированной накачке между компонентами дублета начинает вырастать новая линия, обозначенная на рис. 1 и рис. 5, $a$ как линия $B$, которая при $T=0.5 \mathrm{~K}$ становится абсолютно доминирующей по интенсивности в спектрах. Качественно такое же поведение демонстрируют спектры излучательной рекомбинации, если при фиксированной и самой низкой в эксперименте температуре, $T \approx 0.45 \mathrm{~K}$, увеличивать плотность мощности оптической накачки. Поведение спектров люминесцен- ции в таких условиях демонстрирует рис. 5, $b$. Видно, что новая линия люминесценции (линия $B$ ) становится доминирующей в спектрах при самой низкой температуpe, когда, по нашему предположению, возникает новая конденсированная фаза ТЦМЭ.

Наконец, были выполнены эксперименты, благодаря которым удалось проследить за эволюцией спектров ФЛ при фиксированных температуре $T=0.5 \mathrm{~K}$ и накачке $P=40 \mathrm{~mW} / \mathrm{cm}^{2}$ в условиях плавной развертки магнитного поля. Область развертки магнитного поля $B$ соответствовала вариациям фактора заполнения в пределах $v=2 \pm 0.15$, а, следовательно, изменялась в пределах нескольких процентов и сама величина плотности 2D-электронов. Возникновение нового канала излучательной рекомбинации и доминирование в спектрах соответствующей линии люминесценции $B$ полностью коррелирует с появлением новой фазы ТЦМЭ в полном согласии с фазовой диаграммой, представленной на рис. 4, $c$.

Существенно также подчеркнуть, что вырастающая в спектрах люминесценции новая линия $B$ является полностью циркулярно поляризованной. Ее $\sigma^{+}$циркулярная поляризация и расположение в спектре указывают, что в процессе излучательной рекомбинации с тяжелыми дырками валентной зоны участвуют исключительно электроны верхнего спинового подуровня нулевого уровня Ландау, которые экситонам не принадлежат.

Теперь обсудим возможное происхождение канала излучательной рекомбинации в условиях возникновения новой фазы ТЦМЭ. Можно предположить, что при рождении в исследуемой системе новой экситонной фазы, соответствующей бозе-конденсату, 2D-электроны в зоне проводимости, которые самому экситонному бозе-конденсату непосредственно и не принадлежат, тем не менее косвенно „чувствуют“ появление сильно коррелированного, когерентного квантового состояния и на масштабах пространственной когерентности нового фазового состояния начинают излучательно рекомбинировать с тяжелыми дырками валентной зоны синхронно, в фазе, в прямом соответствии с эффектом Дике [17]. Этот эффект предсказывает сверхлюминесценцию (superradiance) в условиях возникновения когерентности в атомных системах. Именно благодаря этому эффекту линия люминесценции $B$, непрямым образом связанная с возникающей новой фазой ТЦМЭ, становится в конечном итоге самой интенсивной и доминирующей в спектрах ФЛ (см. рис. 5). Существенно, что положение этой линии в шкале энергий не совпадает с какими-либо известными дипольноразрешенными каналами излучательной рекомбинации, которые соответствуют энергетической схеме экспериментально установленных одночастичных (одноэлектронных) состояний (см. схему электронных переходов на рис. 1). Этот факт служит еще одним свидетельством в пользу коллективного происхождения возгорающейся новой линии люминесценции. Само спектральное положение этой линии рассчитать из первых принципов 
пока не удается. Представляется однако, что ее спектральный сдвиг $\approx 70 \mu \mathrm{eV}$ в сторону бо́льших энергий относительно „центра тяжести“ дублета $\sigma^{+}-\sigma^{-}$не случаен и может являться следствием отталкивательного взаимодействия в вырожденной системе взаимодействующих ТЦМЭ, подчиняющихся бозе-статистике. Это может служить косвенным указанием на то, что фазовый переход в 2D-фермиевской системе в условиях фотовозбуждения вырожденного, взаимодействующего бозе-газа магнитоэкситонов несет черты фазового перехода 2-го рода.

\section{4. Бездиссипативное растекание магнетофермионного конденсата и перенос спина на макроскопически большие расстояния}

В выполненных нами экспериментах наблюдалось еще одно, очень интересное и удивительное явление, связанное с поведением времени спиновой релаксации $\tau$ (или времени жизни) триплетных экситонов в низкотемпературной области $T<1 \mathrm{~K}$, где возникает новая конденсированная экситонная фаза. В этой области, точнее непосредственно в области пятна фотовозбуждения, время релаксации ведет себя немонотонно в функции температуры (рис. 4, $b$ ), что может свидетельствовать о не диффузионном характере транспорта экситонов из области возбуждения. Это экспериментальное наблюдение указывает на возможное пороговое изменение вязкости подсистемы неравновесных ТЦМЭ при диффузии таких экситонов к центрам релаксации (рекомбинации). Гипотезу о возможном „сверхдиффузионном“ растекании магнетофермионного конденсата на макроскопически большие расстояния можно проверить экспериментально, используя для этих целей пространственное разнесение световодов, используемых для возбуждения и детектирования собственно конденсированной фазы. Соответствующую схему эксперимента и результат экспериментального наблюдения растекания конденсата на макроскопически большие расстояния из локальной области оптического возбуждения иллюстрирует рис. 6. Согласно приведенной на рисунке схеме, для подвода лазерного света, возбуждающего достаточно плотный ансамбль ТЦМЭ в 2D-фермиевской электронной системе, используется дополнительный третий световод (на рис. 6 слева). Магнитоэкситоны возбуждаются в лазерном пятне с поперечным размером $\sim 0.4 \mathrm{~mm}$ непосредственно под световодом. Два других световода располагаются на расстоянии $\sim 2 \mathrm{~mm}$ от первого (на рис. 6 справа), т.е. достаточно далеко от области возбуждения конденсата, и используются для измерения ФРО, связанного с возникающими ферми-дырками в ТЦМЭ, создаваемыми под удаленным (левым) световодом. Когда температура относительно высока $(T>1 \mathrm{~K})$, то даже при большой плотности фотовозбуждения сигнал

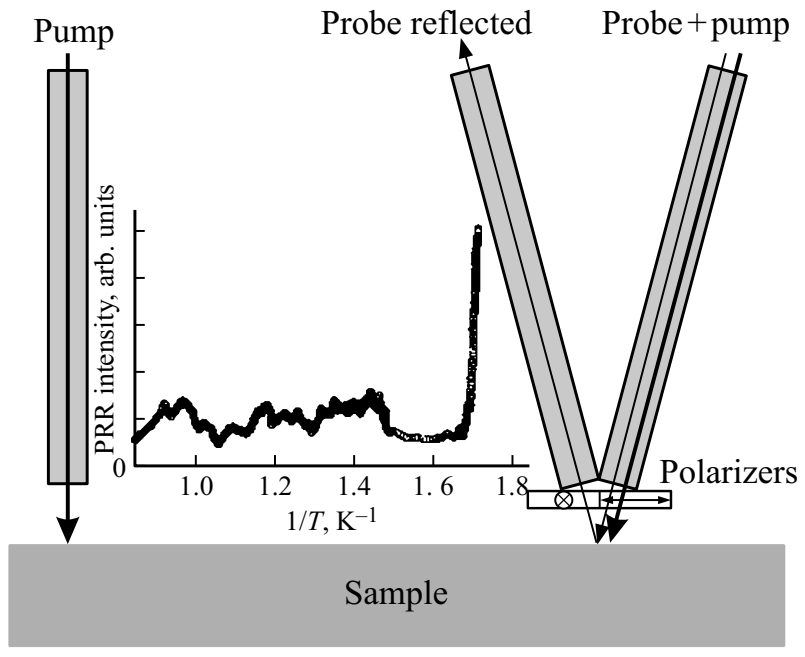

Рис. 6. Схема эксперимента по детектированию растекания магнетофермионного конденсата на макроскопически большие расстояния. Справа - схема обычного двухсветоводного измерения ФРО и ФЛ, слева - дополнительный световод накачки для измерений ФРО с пространственным разделением. Вставка иллюстрирует растекание магнетофермионного конденсата на макроскопические расстояния: сигнал ФРО, детектируемый с помощью пары световодов справа, на расстоянии $\approx 2 \mathrm{~mm}$ от области фотовозбуждения под световодом накачки слева резко возрастает с понижением температуры.

ФРО, связанный с фермиевскими дырками, которые принадлежат ТЦМЭ, ничтожно мал и теряется в шумах, обусловленных остаточным рассеянным светом в системе. Однако при понижении температуры и при той же фиксированной оптической накачке, в области $T \leq 0.75 \mathrm{~K}$, сигнал ФРО пороговым образом гигантски возрастает. Это наблюдение, согласующееся с фазовой диаграммой на рис. 4,c, неопровержимым образом доказывает, что значительная часть фотовозбужденных ТЦМЭ в условиях их конденсации в 2D-фермиевской электронной системе растекается из области возбуждения на макроскопически большие расстояния не диффузионным образом, т.е. бездиссипативно. Такое пороговое изменение диффузионных свойств магнитоэкситонов можно удовлетворительно согласовать с картиной ожидаемой конденсации в пространстве псевдомоментов. Известно, что ТЦМЭ в дипольном приближении не являются оптически активными. Согласно теоретическим работам $[5,6]$, основное состояние 2D-ТЦМЭ по соображениям симметрии должно обладать орбитальными свойствами $p$-состояния и, как следствие, дисперсионная кривая ТЦМЭ в шкале псевдомоментов должна содержать ротонный минимум. В области ротонного минимума магнитоэкситоны обладают псевдомоментом, величина которого обратно пропорциональна магнитной длине $l_{H}$. Благодаря хаотическому флуктуирующему потенциалу, неизбежно присутствующему даже в высокосовершенных гетероструктурах, 
а также при непременном условии достаточно низких температур ТЦМЭ в режиме невырожденного бозе-газа оказываются локализованными на таких флуктуациях, а пространственная ориентация их псевдомоментов в плоскости должна быть случайной. Однако в условиях бозе-вырождения магнитоэкситонов симметрия системы может спонтанным образом нарушаться, а псевдомоменты всех магнитоэкситонов в конденсате выстраиваться при этом вдоль одного направления. Такое выстраивание должно происходить на масштабах крупномасштабной пространственной когерентности.

\section{5. Заключение}

В представленной работе продемонстрировано, что в 2D-электронной фермиевской системе, помещенной в достаточно сильное поперечное магнитное поле, в условиях фотовозбуждения электрически нейтральных, долгоживущих триплетных циклотронных магнитоэкситонов, подчиняющихся бозе-статистике, при достаточно низких температурах и относительно высоких плотностях таких возбуждений возникает качественно новое метастабильное долгоживущее состояние - магнетофермионный конденсат. Конденсированная фаза когерентно взаимодействует с внешним электромагнитным полем и обладает сверхизлучающими свойствами: в ней открывается новый, преобладающий по интенсивности, канал излучательной рекомбинации скоррелированных на масштабах параметра порядка в конденсированной фазе электронов с тяжелыми дырками валентной зоны. Благодаря небольшой вязкости конденсированная фаза демонстрирует бездиссипативное растекание на макроскопически большие расстояния относительно области, где эта фаза непосредственно возбуждается. К настоящему времени отсутствует аналитическое описание собственно конденсированной фазы и связанного с ней параметра порядка. Однако представляется очевидным, что вырожденная часть ТЦМЭ должна сильно взаимодействовать с фермиевской электронной системой, по крайней мере, на масштабах параметра порядка. Скорее всего, это взаимодействие носит обменный характер, однако точный вид гамильтониана этого взаимодействия пока не известен.

Уместно провести сравнение магнетофермионного конденсата с другими, хорошо известными квантовыми объектами, в которых уже давно обнаружено и тщательно исследовано сверхтекучее течение материи. По сравнению с другими объектами магнетофермионный конденсат больше напоминает жидкий ${ }^{3} \mathrm{He}$, который является исключительно фермиевской жидкостью. Сверхтекучесть жидкого ${ }^{3} \mathrm{He}$ ассоциируется с возникновением коррелированных пар фермиевских изотопов атомов ${ }^{3} \mathrm{He}[1]$. Коррелированные пары атомовфермионов являются бозонами, и для них возможна бозе-конденсация. Коррелированные пары ${ }^{3} \mathrm{He}$ являются далеким аналогом куперовских пар в сверхпроводниках. Вместе с тем жидкий гелий - термодинамически равновесная система, тогда как магнетофермионный конденсат в полупроводниковой гетероструктуре метастабилен, и в этом состоит одно из их главных отличий. К тому же, если в случае ${ }^{3} \mathrm{He}$, а также ${ }^{4} \mathrm{He}$ сверхтекучесть связана с бездиссипативным переносом на макроскопически большие масштабы сверхтекучей компоненты самого вещества, то в случае магнетофермионного конденсата на макроскопические расстояния растекается электронное возбуждение и связанный с этим возбуждением результирующий целочисленный спин, привносимый в 2D-электронную систему долгоживущими ТЦМЭ.

Для прояснения микроскопического устройства магнетофермионного конденсата очень существенно выполнить в самое ближайшее время ряд экспериментов. Среди них первостепенное значение имеют исследования пространственной когерентности магнетофермионного конденсата и, в частности, кросс-коррелятора амплитуд 1-го порядка. Эти эксперименты должны выполняться в духе классических опытов Юнга, хорошо известных в волновой оптике. В интересующем нас случае в результате интерференционного сложения на масштабах пространственной когерентности должна возникать картина гармонической модуляции электронной плотности в самом конденсате. Интересно также измерить скорость распространения самого возбуждения, связанного с бозе-конденсатом ТЦМЭ. Очень важно знать, насколько эта скорость отличается от скорости звука. Наконец, большой интерес представляют исследования электронного транспорта в присутствии магнетофермионного конденсата в двумерном фермиевском газе.

Настоящая статья приурочена к дню памяти Евгения Федоровича Гросса - выдающегося отечественного физика, первопроходца и первооткрывателя такого замечательного явления, как водородоподобный экситон.

\section{Список литературы}

[1] A.J. Leggett. Rev. Mod. Phys. 71, S318 (1999).

[2] G.D. Mahan. Phys. Rev. 163, 612 (1967).

[3] P. Nozières, C.T. De Dominicis. Phys. Rev. 178, 1097 (1969).

[4] X. Cui, C. Wang, A. Argondizzo, S. Garrett-Roe, B. Gumhalter, H. Petek. Nature Phys. 10, 505 (2014).

[5] Ю.А. Бычков, С.В. Иорданский, Г.М. Элиашберг. Письма в ЖЭТФ 33, 152 (1981).

[6] C. Kallin, B.I. Halperin. Phys. Rev. B 30, 5655 (1984).

[7] J.K. Jain. Phys. Rev. Lett. 63, 199 (1989); Adv. Phys. 41, 105 (1992).

[8] S. Dickmann, I.V. Kukushkin. Phys. Rev. B 71, 241310 (2005).

[9] L.V. Kulik, I.V. Kukushkin, S. Dickmann, V.E. Kirpichev, A.B. Van'kov, A.L. Parakhonsky, J.H. Smet, K. von Klitzing, W. Wegscheider. Phys. Rev. B 72, 073304 (2005). 
[10] S. Dickmann. Phys. Rev. Lett. 110, 166801 (2013).

[11] И.В. Лернер, Ю.Е. Лозовик. ЖЭТФ 80, 1488 (1981).

[12] L.V. Kulik, A.V. Gorbunov, A.S. Zhuravlev, V.B. Timofeev, S. Dickmann, I.V. Kukushkin. Sci. Rep. 5, 10354 (2015).

[13] W. Kohn. Phys. Rev. 123, 1242 (1961).

[14] Q. Zhang, T. Arikawa, E. Kato, J.L. Reno, W. Pan, J.D. Watson, M.J. Manfra, M.A. Zudov, M. Tokman, M. Erukhimova, A. Belyanin, J. Kono. Phys. Rev. Lett. 113, 047601 (2014).

[15] L.V. Kulik, S. Dickmann, I.K. Drozdov, A.S. Zhuravlev, V.E. Kirpichev, I.V. Kukushkin, S. Schmult, W. Dietsche. Phys. Rev. B 79, 121310 (2009).

[16] L.V. Kulik, A.S. Zhuravlev, S. Dickmann, A.V. Gorbunov, V.B. Timofeev, I.V. Kukushkin, S. Schmult. Nature Commun. 7, 13499 (2016).

[17] R.H. Dicke. Phys. Rev. 93, 99 (1954).

Редактор Ю.Э. Китаев 